\title{
Multiband Fractal Patch Antenna: Modification and Miniaturization of Sierpinski Gasket
}

\author{
Athira .P1, V. P. Joseph ${ }^{2}$ \\ ${ }^{1}$ SCMS School of Engineering and Technology Karukutty, Kerala \\ ${ }^{2}$ Department of Physics, Christ College Irinjalakuda, Kerala
}

\begin{abstract}
The evolution of the current systems using fourth generation technology is based on the key idea of interoperability. In future systems, a single terminal should be capable of performing all the functions - anywhere, at any moment and in any interface, resulting in a convergence of services. This paper presents the design and simulation of a novel miniaturized microstrip fractal patch antenna which resonates at $3.1 \mathrm{GHz}, 3.8 \mathrm{GHz}, 5.6 \mathrm{GHz}, 6.9 \mathrm{GHz}$ and $7.7 \mathrm{GHz}$.The size of the antenna is compacted by $30 \%$ in comparison with a regular Sierpinski gasket antenna. All the simulations have been performed using ANSYS HFSS software. The obtained results have also been validated using Keysight $N 9915 A$ network analyzer. The simulated and experimental results are found to be in good agreement.
\end{abstract}

Keywords: interoperability, miniaturization, fractal antenna, Sierpinski gasket

\section{Introduction}

Fractal antenna technology has been a recent topic of interest. Antennas are important component of any device and therefore the space occupied by them in any device is a subject of concern. There has been a tremendous amount of improvement in every field of technology and size of almost all devices has reduced considerably. But antenna has not faced a similar evolution. The size reduction that can be brought to an antenna is highly limited. And this is the major bottleneck faced by any antenna designer. In such a situation, a fractal antenna can act as a good candidate satisfying its designer with desired performance. Traditional mobile phones have been replaced by smart phones with a wide range of applications like Bluetooth, Wi-Fi, WiMAX etc. If we are to design separate antennas for each of these applications the device may look like a porcupine. Hence, multiband property is very essential to ensure interoperability. And here comes the importance of fractal antennas which can provide a good multiband operation. Compact sizes, low profile, conformal and multiband are the highly desirable attributes of a microstrip patch antenna .The term ,fractale, which means broken or irregular fragments, was originally coined by Mandelbrot to describe a family of complex shapes that possess an inherent self-similarity or self affinity in their geometrical structure. Many analysis works have been performed in the field of multiband fractal antennas which focuses on the construction of Sierpinski gasket [1]-[9] and Sierpinski carpet [10]-[13]. Fractal antennas are mainly used for miniaturization and multiband operations. Accordingly, they are classified as small size antennas (e.g.; Hilbert curve, Minkowski loops etc) and multiband antennas (e.g.; Sierpinski gasket and Sierpinski carpet).The design of a $2.45 \mathrm{GHz}$ triangular fractal antenna using fractal geometry is presented in [3]. It is observed that with increase in the number of iterations the bandwidth of the antenna increases and in second and third iterations the antenna starts showing multiband behavior. It is also demonstrated that the majority of the self-similar fractal gap structure can be eliminated from the Sierpinski gasket without significantly affecting its multiband behavior [8]. The proposed paper discusses about the design and simulation of a Sierpinski gasket antenna, its modified structure and its miniaturized structure.

\section{Multiband Behavior of Sierpinski Gasket}

The multiband property of Sierpinski gasket is analyzed in this section. Figure 1 shows the patches of a Sierpinski gasket for each iteration and figure 2 depicts the fabricated antenna for the third iteration.
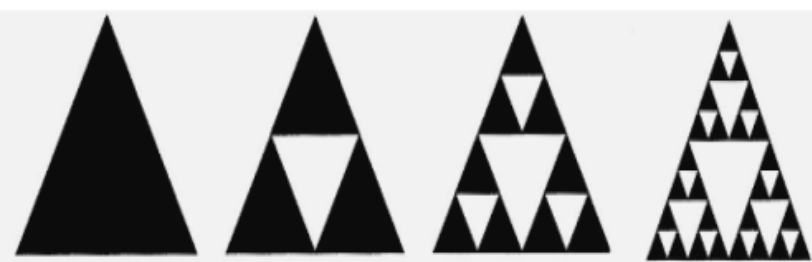

Figure 1: Sierpinski gasket: basic structure, first Iteration, second iteration and third iteration

The overall size of the antenna is $80 \mathrm{~mm} \times 83 \mathrm{~mm}$. A Sierpinski gasket is designed at $1.9 \mathrm{GHz}$ with one side of the triangular patch measuring $64 \mathrm{~mm}$ in dimension. All the designs corresponding to the three iterations were fabricated. The height of the substrate is $1.6 \mathrm{~mm}$ and the dielectric constant chosen is 3.38 .

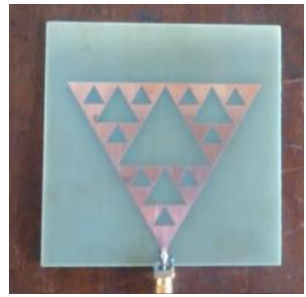

Figure 2: Fabricated Sierpinski gasket for third iteration

The resonant frequency $f_{r}$ can be determined by,

\section{Volume 4 Issue 12, December 2015}




\section{International Journal of Science and Research (IJSR) \\ ISSN (Online): 2319-7064 \\ Index Copernicus Value (2013): 6.14 | Impact Factor (2014): 5.611}

$$
\begin{aligned}
& f_{r}=2 c /\left(3 L_{\text {eff }} \sqrt{ } \epsilon_{r}\right) \\
& L_{\text {eff }}=\sqrt{ } 3 \cdot a / 2
\end{aligned}
$$

where, „ $\mathrm{L}_{\mathrm{eff}}{ }^{\text {ee }}$ is the effective length of the equivalent rectangular patch, , $\mathrm{a}^{\mathrm{ee}}$ is the side of the triangular patch and $\epsilon_{\mathrm{r}}$ ,the relative permittivity. Ideal fractal geometry concepts cannot be used in designing antennas and therefore only up to the 3 rd order of the fractal antenna is investigated. The $S_{11}$ plots for the different iterations are shown in figures $3,4,5$ and 6.

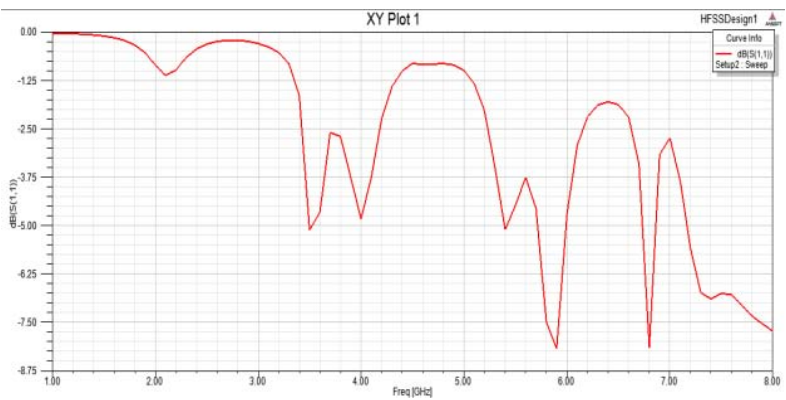

Figure 3: $\mathrm{S}_{11}$ plot for the basic structure

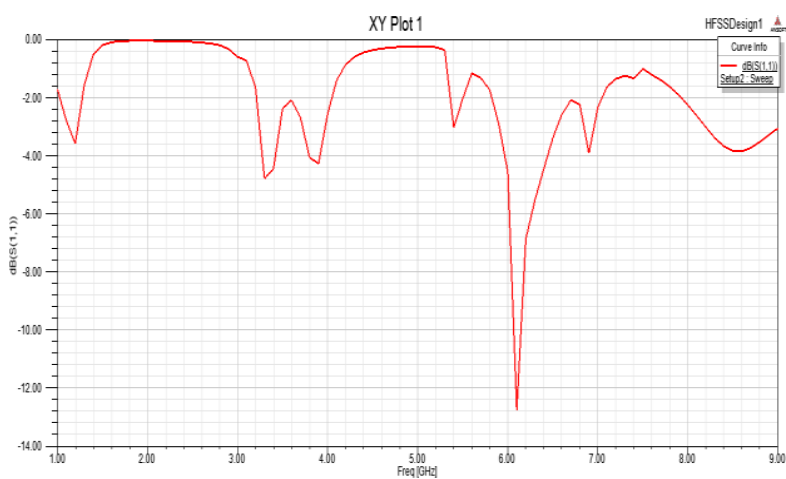

Figure 4: $\mathrm{S}_{11}$ plot for the first iteration

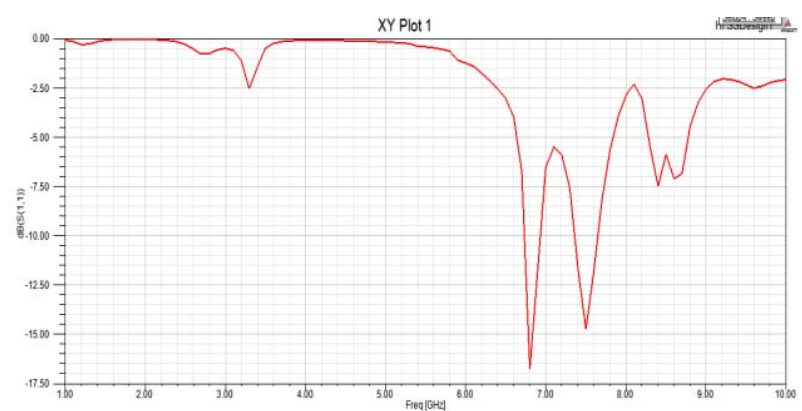

Figure 5: $\mathrm{S}_{11}$ plot for second iteration

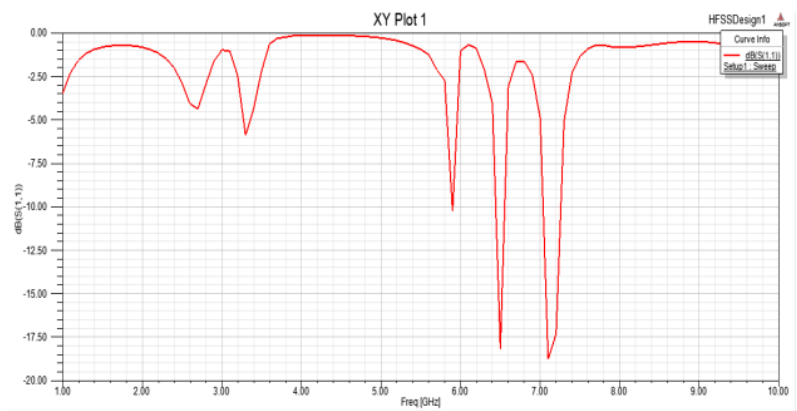

Figure 6: $S_{11}$ plot for the third iteration

For the base geometry, no radiating bands with considerable gain was identified, where as for the first iteration a radiating band at $6.1 \mathrm{GHz}$ with a return loss of $-12.71 \mathrm{~dB}$ was observed. As we can see, for the second iteration, two bands at $6.8 \mathrm{GHz}$ and $7.5 \mathrm{GHz}$ with $-16.72 \mathrm{~dB}$ and $-14.71 \mathrm{~dB}$ return losses respectively were observed and for the third iteration the antenna shows resonances at $5.9 \mathrm{GHz}, 6.5 \mathrm{GHz}$ and $7.1 \mathrm{GHz}$ with a return loss of $-10.24 \mathrm{~dB},-18.12$ and $-18.71 \mathrm{~dB}$ respectively. The observed values are in quite agreement with experimental results and are tabulated in Table 1.

Table 1: Comparison of theoretical and experimental values for resonant frequency, return loss and bandwidth for all the three iterations

\begin{tabular}{|c|c|c|c|c|c|c|}
\hline \multirow{2}{*}{ Iteration } & \multicolumn{2}{|c|}{$\begin{array}{c}\text { Resonant Frequency } \\
(G H z)\end{array}$} & \multicolumn{2}{c|}{$\begin{array}{c}\text { Return Loss } \\
(d B)\end{array}$} & \multicolumn{2}{c|}{$\begin{array}{c}\text { Bandwidth } \\
(\text { GHz })\end{array}$} \\
\cline { 2 - 7 } & Theoretical & Experimental & Theoretical & Experimental & Theoretical & Experimental \\
\hline 0 & 5.9 & 7.5 & -8 & -8.2 & 0.12 & 0.1 \\
\hline 1 & 6.1 & 5 & -10.2 & -14.11 & 0.25 & 0.2 \\
\hline 2 & 6.8 & 6.6 & -17 & -14.74 & 0.35 & 0.4 \\
& 7.5 & 7.76 & -14.5 & -14.41 & 0.45 & 0.4 \\
\hline 3 & 5.9 & 5.59 & -11 & -17.89 & 0.2 & 0.5 \\
& 6.5 & 6.2 & -18.25 & -10.37 & 0.3 & 0.4 \\
& 7.1 & 8.68 & -18.75 & -18.79 & 0.4 & 0.6 \\
\hline
\end{tabular}

From these results, the following conclusions can be made:

- The resonant frequency increases with increase in the number of iterations

- The multiband behavior is obtained as the numbers of iterations are increased

- The return losses improve as the number of iterations increase

- The bandwidth of the antenna gets increased too with increase in the number of iterations

\section{Modified Sierpinski Gasket}

A modified Sierpinski gasket was designed and simulated conceiving the idea from a regular Sierpinski gasket. The analysis presented here demonstrates that the multiband behavior of Sierpinski gasket is primarily a function of the periodic placement of the four gaps located along the central vertical axis of the antenna. It is demonstrated that the majority of the self-similar fractal gap structure can be eliminated from the Sierpinski gasket without significantly affecting its multiband behavior [8]. Hence all other gaps except those which run through the central vertical axis can 


\section{International Journal of Science and Research (IJSR) \\ ISSN (Online): 2319-7064 \\ Index Copernicus Value (2013): 6.14 | Impact Factor (2014): 5.611}

be removed. It is interesting to note that for each successive operating band, the current distribution through the Sierpinski gasket truncates at the extent of the sub gasket corresponding to each iteration [1],[2]. From this truncation of the current distribution, it seems reasonable to conclude that the major gap structures located at the extent of each sub gasket primarily determines the multiband behavior of the Sierpinski gasket. Evidence of this is obtained by eliminating the lesser gap structure within each sub gasket as shown in figure 7.

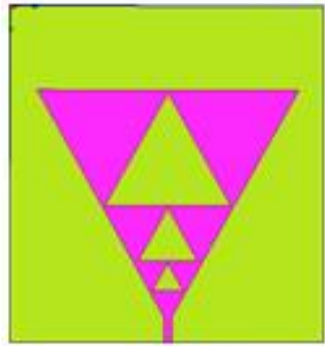

(a)

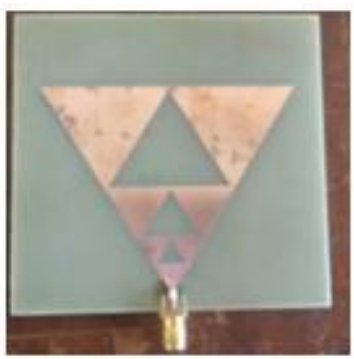

(b)
Figure 7: Modified Sierpinski Gasket (a) Schematic view (b) Fabricated structure

The $\mathrm{S}_{11}$ result for the modified structure is shown in figure 8 . As one can see the two resonating bands at $6.7 \mathrm{GHz}$ and $7.6 \mathrm{GHz}$ remain the same for the modified structure. In addition to the two bands, a new band has been introduced for the modified structure at $3.6 \mathrm{GHz}$ with a return loss of $-10.23 \mathrm{~dB}$. The appearance of the new band at $3.6 \mathrm{GHz}$ is at the cost of disappearance of the band at $5.9 \mathrm{GHz}$. Also, $3.6 \mathrm{GHz}$ is a more useful frequency value when compared to $5.9 \mathrm{GHz}$.

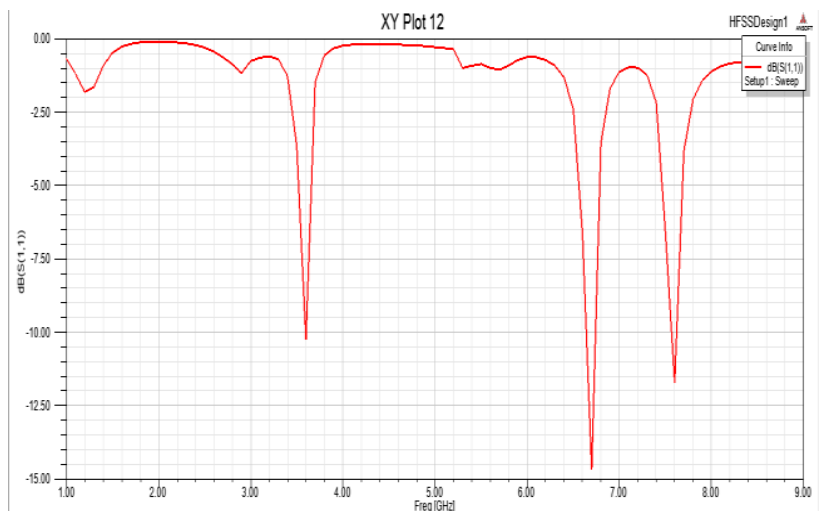

Figure 8: $\mathrm{S}_{11}$ plot for modified structure (simulated)

Figure 9 shows the experimental curve for the modified structure. A Keysight N9915A Vector Network Analyzer is used for the experimental study. This clearly explains the significance of the air gaps through the central vertical axis in determining the multiband behavior. The three air gaps through the central vertical axis are necessary to provide multiband. This means that we can equally end up in the modified structure given in figure 7; which can provide us with the similar results as that of a regular Sierpinski gasket.

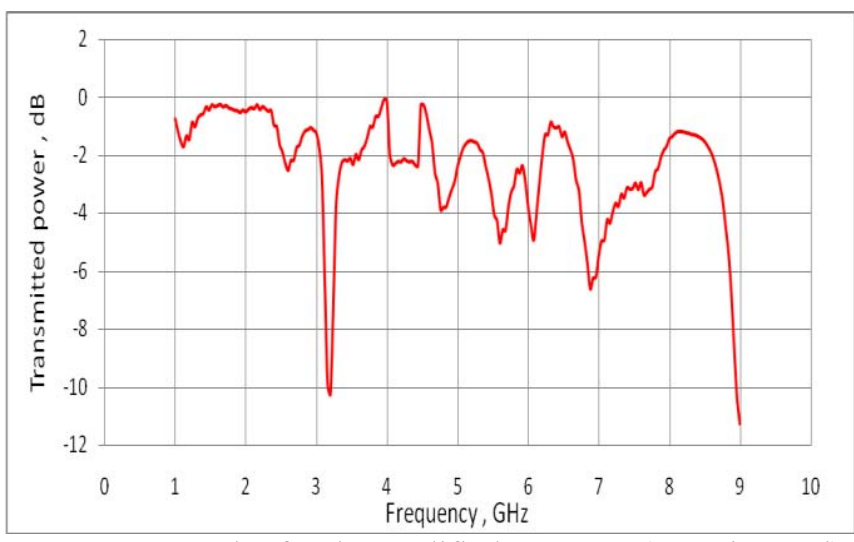

Figure 9: $\mathrm{S}_{11}$ plot for the modified structure (experimental)

\section{Miniaturization}

A miniaturized structure of the modified Sierpinski fractal antenna is presented. It is designed by conceiving the ideas and results of a Sierpinski gasket structure and it's modified one. From the previously simulated structures we can conclude that the gaps through the central vertical axis of the structure play an important role in positioning of the resonating bands. The overall height of the triangle is considerably reduced. The gaps have been placed so as to get the same results as that of the previous structure. At the top of the patch a trapezoidal gap is provided in order to force the current to flow through a convoluted path [9]. The other gaps are provided in the shape of a triangle. This gap can be of any shape; but a triangle serves as the well suited one as it has pointing edges. Each vertex of the air gap perturbs into the current that is mainly concentrated at edges of the structure. Figure 10 shows the experimental $\mathrm{S}_{11}$ plot for the modified Sierpinski gasket. The overall dimension of the design is $50 \mathrm{~mm} \times 53 \mathrm{~mm}$ with a substrate height of $1.6 \mathrm{~mm}$ and a relative permittivity of 3.38 . The dimension of the ground is $50 \mathrm{~mm} \times 40.7 \mathrm{~mm}$. A miniaturization of about $30 \%$ has been achieved.

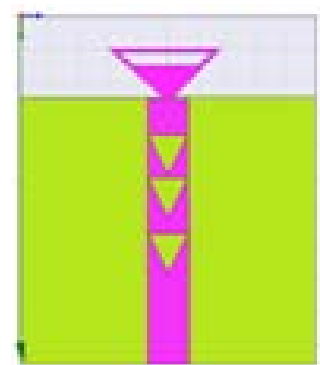

(a)

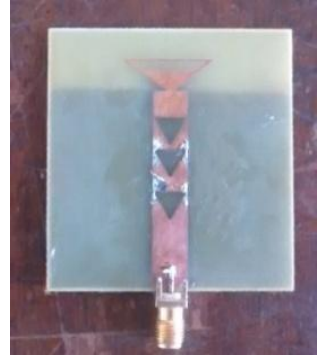

(b)
Figure 10: Miniaturized fractal antenna (a) Schematic view (b) Fabricated structure

The simulated and experimental S11 plots for the modified miniaturized fractal antenna are shown in figure 11 and figure 12 respectively. 


\section{International Journal of Science and Research (IJSR) \\ ISSN (Online): 2319-7064}

Index Copernicus Value (2013): 6.14 | Impact Factor (2014): 5.611

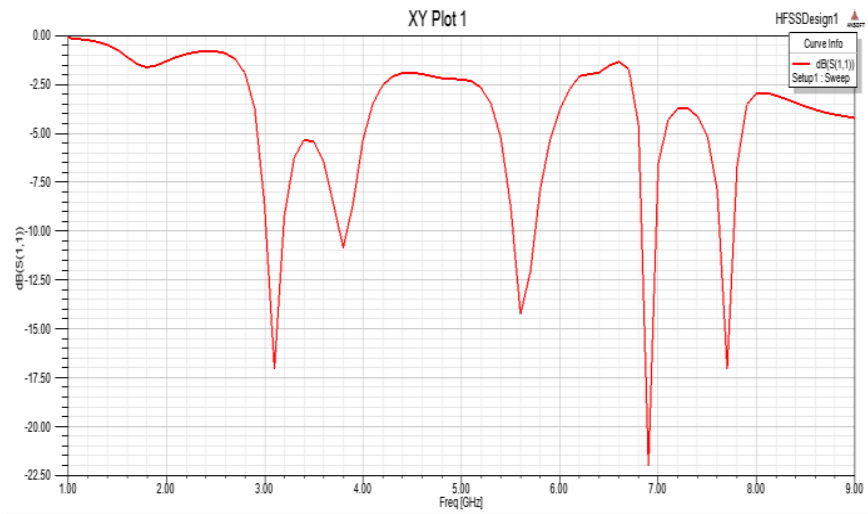

Figure 11: $\mathrm{S}_{11}$ plot for the miniaturized structure (simulated)

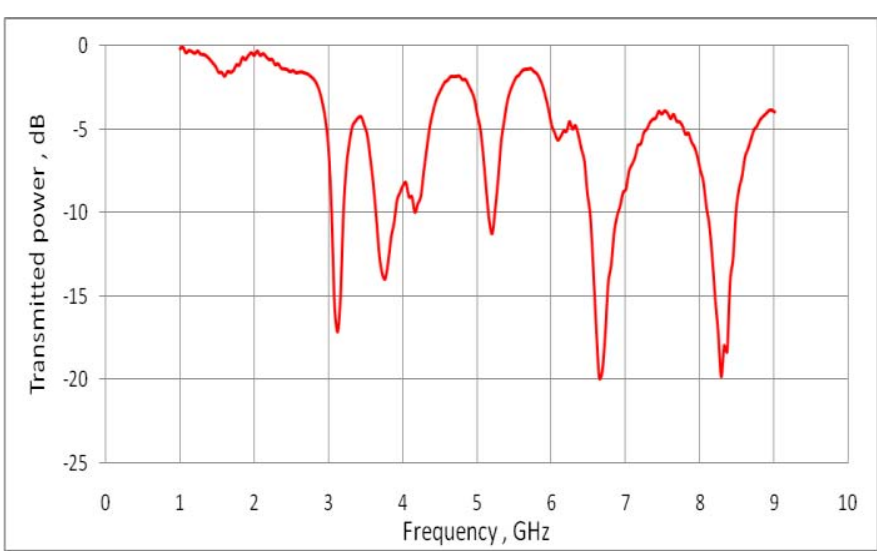

Figure 12: $\mathrm{S}_{11}$ plot for miniaturized structure (experimental)

The antenna resonates at 5 frequencies, namely $3.1 \mathrm{GHz}$, $3.8 \mathrm{GHz}, 5.6 \mathrm{GHz}, 6.9 \mathrm{GHz}$ and $7.7 \mathrm{GHz}$. The second and third resonating bands $(3.8 \mathrm{GHz}$ band and $5.6 \mathrm{GHz}$ band) have wide bandwidth compared to the other bands. Table 2 describes comparison of theoretical and experimental values for resonant frequency return loss and bandwidth for the modified and miniaturized structure.

Table 2: Comparison of theoretical and experimental values for resonant frequency, return loss and bandwidth for the modified and miniaturized structure

\begin{tabular}{|c|c|c|c|c|c|c|}
\hline \multirow{2}{*}{} & \multicolumn{2}{|c|}{$\begin{array}{c}\text { Resonant Frequency } \\
(\mathrm{GHz})\end{array}$} & \multicolumn{2}{c|}{$\begin{array}{c}\text { Return Loss } \\
(d B)\end{array}$} & \multicolumn{2}{c|}{$\begin{array}{c}\text { Bandwidth } \\
(\mathrm{GHz})\end{array}$} \\
\cline { 2 - 7 } & Theoretical & Experimental & Theoretical & Experimental & Theoretical & Experimental \\
\hline Modified Structure & 3.6 & 3.1 & -10.5 & -11.5 & 0.3 & 0.2 \\
& 6.7 & 6.89 & -14.75 & -10 & 0.3 & 0.5 \\
& 7.6 & 8.99 & -11.75 & -14.01 & 0.4 & 0.5 \\
\hline Miniaturized structure & 3.1 & 3.1 & -17.5 & -18.26 & 0.2 & 0.3 \\
& 3.8 & 3.75 & -10.5 & -16.08 & 0.5 & 0.4 \\
& 5.6 & 5.18 & -14.5 & -12.69 & 0.5 & 0.4 \\
& 6.9 & 6.64 & -22 & -22.09 & 0.2 & 0.5 \\
& 7.7 & 8.27 & -17 & -23.08 & 0.2 & 0.5 \\
\hline
\end{tabular}

In order to understand which part of the antenna is being utilized at each resonance, the surface current densities are analyzed for each of the resonant frequencies. This gives an intuitive insight as to how the antenna is operating at multiple frequencies. The current flow is forced in a way of concentration at the subscale version of the antenna elements, having a perimeter comparable to the wavelength. This helped in positioning of the air gaps.

\section{Conclusion}

This paper presents the design of a multiband Sierpinski gasket and its modified structure where major portions of the Sierpinski gasket's self-similar fractal gap structure were altered or eliminated completely. A miniaturized fractal antenna was designed and fabricated using the concepts of Sierpinski gasket. A miniaturization of $30 \%$ was observed. It was concluded that the geometry of Sierpinski gasket is fully determined by height, flare angle, iteration number, and scaling factor. Positions of different bands can be controlled by proper adjustment of the above factors. All the simulation results have been supported by the corresponding experimental results. The proposed design provides a high degree of flexibility in choosing the number of bands and the associated band spacing for a candidate antenna design.

\section{References}

[1] C. Puente, J. Romeu, R. Pous, X. Garcia and F. Benitez, "Fractal multiband antenna based on the Sierpinski gasket", Electronics Letters 4th January, vol 32 No.1, IEEE, 1996.

[2] M.Navarro, J.M.Gonzalez, C.Puene, J.Romeu and A.Aguasca, "Self-similar Surface Current Distribution on Fractal Sierpinski Antenna Verified with Infra-red Thermograms", IEEE, 1999.

[3] L. Yadava, M. Ram and S. Das, "Multiband Triangular Fractal Antenna for Mobile Communication", International Journal of Engineering Science and Technology, vol.2 (11), 6335-6348, 2010.

[4] Muhammad Waqas, Zubair Ahmed, and Mojeeb Bin Ihsan, "Multiband Sierpinski Fractal Antenna", INMIC, IEEE, 2009.

[5] Basil K Jeemon, Shambavi K, Zachariah C Alex, "Design and Analysis of a Multi-fractal Antenna For UWB Application", IEEE International Conference On Emerging Trends In Computing, Communication And Nanotechnology (ICECCN) 2013.

[6] Xinbo Qu , Zhaoxiang He,Wei Shi1, Yunzhi Liu and Zuping Qian "Multiband Analysis of Sierpinski Gasket Antenna with An Iterative Method", International 
Conference on Wireless Communications and Signal Processing, IEEE, 2009

[7] Monika singh, Navneet kumar, Santanu Diwari and, Pradyot Kala, "Bandwidth Enhancement using Small Triangles on Sierpinski Fractal", International Conference on Signal Processing and Communication, IEEE, 2013.

[8] Steven R. Best, "On the Significance of Self-similar Fractal Geometry in Determining the Multiband Behavior of the Sierpinski Gasket Antenna" Antennas And Wireless Propagation Letters, vol. 1, IEEE, 2002.

[9] Wojciech J. Krzysztofik , "Modified Sierpinski Fractal Monopole for ISM-Bands Handset Applications", IEEE Transactions on Antennas and Propagation, Vol. 57, No. 3, March 2009.

[10] Sika Shrestha, Jung-Jin Park, Sun-kuk Noh,and DongYou Choi, "Design of $2.45 \mathrm{ghz}$ Sierpinski fractal based Miniaturized Microstrip Patch Antenna", APCC,IEEE, 2012.

[11]M.K.A.Rahim, N.Abdullah and M.Z.A Abdul aziz, "Microstrip Sierpinski Carpet Antenna Design", APCC, IEEE, 2005.

[12] Yao Na and Shi Xiao-wei, "Analysis of the Multiband Behavior on Sierpinski Carpet Fractal Antennas", APMC Proceedings, IEEE, 2005.

[13]R.Mohanamurali and T.Shanmugananham, "Sierpinski Carpet Fractal Antenna for Multiband Applications", International journal of computer applications, volume 39-no.14, February 2012.

\section{Author Profile}

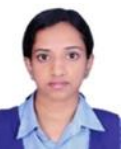

Athira. $\mathbf{P}$ received B.Tech in Electronics and Communication from $\mathrm{MET}^{\mathrm{ee}} \mathrm{S}$ School of Engineering, University of Calicut, in 2013 and M.Tech in Communication Engineering from SCMS School of Engineering and Technology, M.G University, Kottayam in 2015.

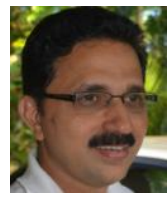

V.P. Joseph obtained M.Sc. in Physics from St. Thomas College Pala 1886 and Ph.D. from Cochin University of Science and Technology in 2000. Presently he is working as Associate Professor in the Research Department of Physics, Christ College, Irinjalakuda. 